\title{
Cluster based Multipath Routing for Wireless Multimedia Sensor Networks
}

\author{
Ranjith Kumar Anandan ${ }^{1 *}$ \\ Sivagami Amirthalingam² \\ ${ }^{\text {I} S a t h y a b a m a}$ University, Chennai, India \\ ${ }^{2}$ Asan Memorial College of Engineering and Technology, Chengalpet, Chennai, India \\ *Corresponding author's Email: ranjithkumara0205@gmail.com
}

\begin{abstract}
Wireless Multimedia Sensor Network is extensively used in the transmission of the multimedia data such as video traffic, etc. This multimedia traffic is transmitted through large distances and hence cluster based multipath routing is widely used. The cluster based routing effectively routes the traffic through the network towards the destination. But, due to the heavy load on the cluster head because of the extensive cluster operation, the energy consumed by the cluster head increases, eventually leading to the death of the cluster head which affects the entire cluster and thus degrades the network operation. So, in this paper, we propose an efficient cluster based multipath routing technique in which each cluster consists of two cluster heads, one to collect cluster data and the other to transmit data. In this way, the overall load on the cluster head is shared between the two cluster head and this increases the cluster lifetime and efficiency. Then the data to be sent is prioritized and then transmitted through the corresponding priority disjoint paths to ensure successful delivery of the data as per the real time requirement.
\end{abstract}

Keywords: Wireless Sensor Network; multipath routing; traffic; efficiency.

\section{Introduction}

\subsection{Wireless Multimedia Sensor Networks}

In the Wireless Sensor Networks (WSN), there exist several sensor nodes which are capable of sensing and routing. This enhanced ability of WSN allows effective data transmission. The transmission of multimedia data through WSN is extensively researched and widely used in multimedia surveillance, storage of data from networked cameras, traffic situations, collision avoidance, etc. However, the multimedia data transmission is prone to limitations because of restricted bandwidth, delay intolerance, specific path features, low power supply.

Hence, the Wireless Multimedia Sensor Network (WMSN) is developed which has characteristics like minimum expense, small sized sensor nodes, ability to transmit video/ audio traffic, and also the special features of WSN. Some of the explicit features of WMSN are increased bandwidth, QoS needs, lower consumption of power, node movement. In WMSN, based on the application, the QoS needs are determined and accordingly mobility of the sensor nodes is minimized. The needs of different types of multimedia traffic like images, video, audio and scalar data are different. Some of the applications of WMSN are in broadband wireless networks, satellite networks, MANET, etc [1, 2].

\subsection{Multipath Routing in WMSN}

The presence of multiple paths leads to multipath routing and because of the enhanced multipath routing algorithms, data transmission through multiple routes has become practical. Multipath routing has several advantages like it makes data transmission more reliable, balances the energy usage, balances load on the nodes, makes the network tolerant to errors, makes network dynamic, and handles bandwidth utilization appropriately. Due to the availability of greater bandwidth, the end to end delay is minimized $[3,4,5,6]$. In building disjoint paths between the source and sink node, the base station is considered as the base of the network, and path from source to sink is developed based on the $\mathrm{N}$ to 1 multipath load balancing routing technique. So, in case of any issue in the main path, back up paths are used [4]. In WSN, multipath routing offers reliability and in WMSN, the multipath routing enables transmission of huge 
multimedia data. In WMSN, packet loss issue is also considered [5].

In the current technologies, delay and loss at the sink is not taken into account while developing multipath routing. This results in degradation of the resource energy and eventually leads to increased overhead due to usage of numerous paths [2].

\subsection{Clustering in WMSN}

Extensive research is being carried out in the field of Hierarchical (clustering) technology. In this technology, a set of sensor nodes are collectively referred as a cluster and one node is chosen to become the cluster head. The data collected by the sensor nodes are transmitted to the cluster head. Since the data collected by the nodes of the same cluster tend to be similar and so the cluster head collects all the data and eliminates the repeating data and then finally transmits a single data packet to the sink node. The data packet is sent to the sink through several paths from the cluster head [6].

In the traditional hierarchical routing technique, the cluster head is supposed to directly communicate with the sink. Hence, clusters are not used in huge networks [6].

In this paper, we propose to define a new cluster based multipath routing for wireless multimedia sensor networks with increased throughput, high packet delivery ratio, decreased time delay, energy consumption etc. In the proposed protocol, two types of cluster heads: main cluster heads and vice cluster heads are used in a cluster so that data collection and fusion is done by main cluster head and data transmission of data to outside. Main cluster heads are chosen based on load and link quality in addition to the residual energy. The vice cluster heads are chosen by the main cluster heads when main cluster heads are overloaded or failed and are used to forward the data. These vice cluster heads unload part of energy consumption of main cluster heads. The rest of the paper is organized as follows. Section II provides various researches conducted in relation to our proposed work. Section III explains about the design strategy and the proposed method. Section IV shows the result and discussion of our proposed method and finally section $\mathrm{V}$ concludes the proposed method

\section{Literature Review}

J. Agrakhed et al [1] have presented a cluster based QoS Adaptive Multipath routing in WMSN. In this routing technique, several paths are selected based on quality metric, cost functions and so factors like data loss rate, energy level and transmission delay are monitored thoroughly. The path which fulfills the routing constraint most efficiently is chosen for data transmission and the importance given to each factor is handled accordingly.

Q. Ye et al [5] have presented a packet scheduling technique for disjoint multipath routing based wireless multimedia sensor networks. In this technique, the data packets are classified into four categories based on its real time as well as its reliability needs, and then priority is allocated to each category. In the traffic scheduling algorithm, non preemptive queuing model with traffic allocation models based on several priority levels and routing path constraints are used.

H. Huang et al [7] have proposed a generalized QoS-aware routing model based on multiple routing metrics and priorities of packets. Initially, IPACR alforithm which is basically a $2 \mathrm{D}$ plain-based routing algorithm is used to improve the conventional ant colony algorithm. The initial allocation of the pheromone is optimized to increase the convergence rate of the algorithm. For larger networks, ICACR algorithm is used which is basically a clustering based routing algorithm and in this algorithm, the iteration time is larger.

S. Lohier et al [8] have proposed a cross-layer routing protocol to handle the multimedia traffic in the hierarchical and heterogeneous networks by using a cost function. The cost of the routing procedure and needs for the multimedia functioning in order to achieve soft quality of service (Soft QoS) is robustly determined by the protocol. To utilize the physical and MAC features, the cross layer mechanism is deployed during routing. But, the throughput attained using this protocol is low.

Y. Sun et al [9] have proposed a QoS routing protocol and an ant-based service-aware routing (ASAR) algorithm for multimedia sensor networks. Various QoS needs of several services are satisfied by this algorithm, by choosing appropriate paths such that the network resource is used completely and performance is enhanced.

$X$. Li et al [10] have proposed an energyefficient routing protocol based on particle swarm clustering algorithm and inter-cluster routing algorithm for WSN. This protocol achieves steady data transmission, saves energy as well as balances load between various nodes. This protocol overcomes the clustering issue but renders low throughput.

Md. A. Alim et al [11] have proposed a fuzzy logic based energy-aware dynamic clustering scheme to extend the network lifetime in terms of

International Journal of Intelligent Engineering and Systems, Vol.9, No.4, $2016 \quad$ DOI: 10.22266/ijies2016.1231.09 
LND. In the fuzzy interference system, two inputs are applied and based on the fuzzy system output, the cluster head is selected. Also, lower load and lesser complexity is achieved by this protocol.

F. Li et al [12] have proposed an Energy-aware Data Gathering Protocol on the basis of the Double Cluster-heads protocol (EAGRDC). In the EAGRDC protocol, based on the comparison between the sensor node's residual energy and average residual energy of the node, the cluster head is chosen. After the selection of the main cluster head, a vice cluster head is chosen to perform the task of data transmission. Thus the overall load on the main cluster head is reduced.

J. Peng et al [13] have proposed an energyefficient prediction clustering algorithm, for heterogenous sensor network. In this algorithm, the selection of the cluster head is performed on the basis of the energy and communication cost. As a result, for a node, its chances of becoming the cluster head is higher if its residual energy is larger. Hence, the energy usage in the network remains balanced.

\section{Cluster based Multipath Routing for Wireless Multimedia Sensor Networks}

\subsection{Overview}

In this paper, we propose to define a new cluster based multipath routing for wireless multimedia sensor networks with increased throughput, high packet delivery ratio, decreased time delay, energy consumption etc. In the proposed protocol, two types of cluster heads: main cluster heads and vice cluster heads are used in a cluster so that data collection and fusion is done by main cluster head and data transmission of data to outside [12].

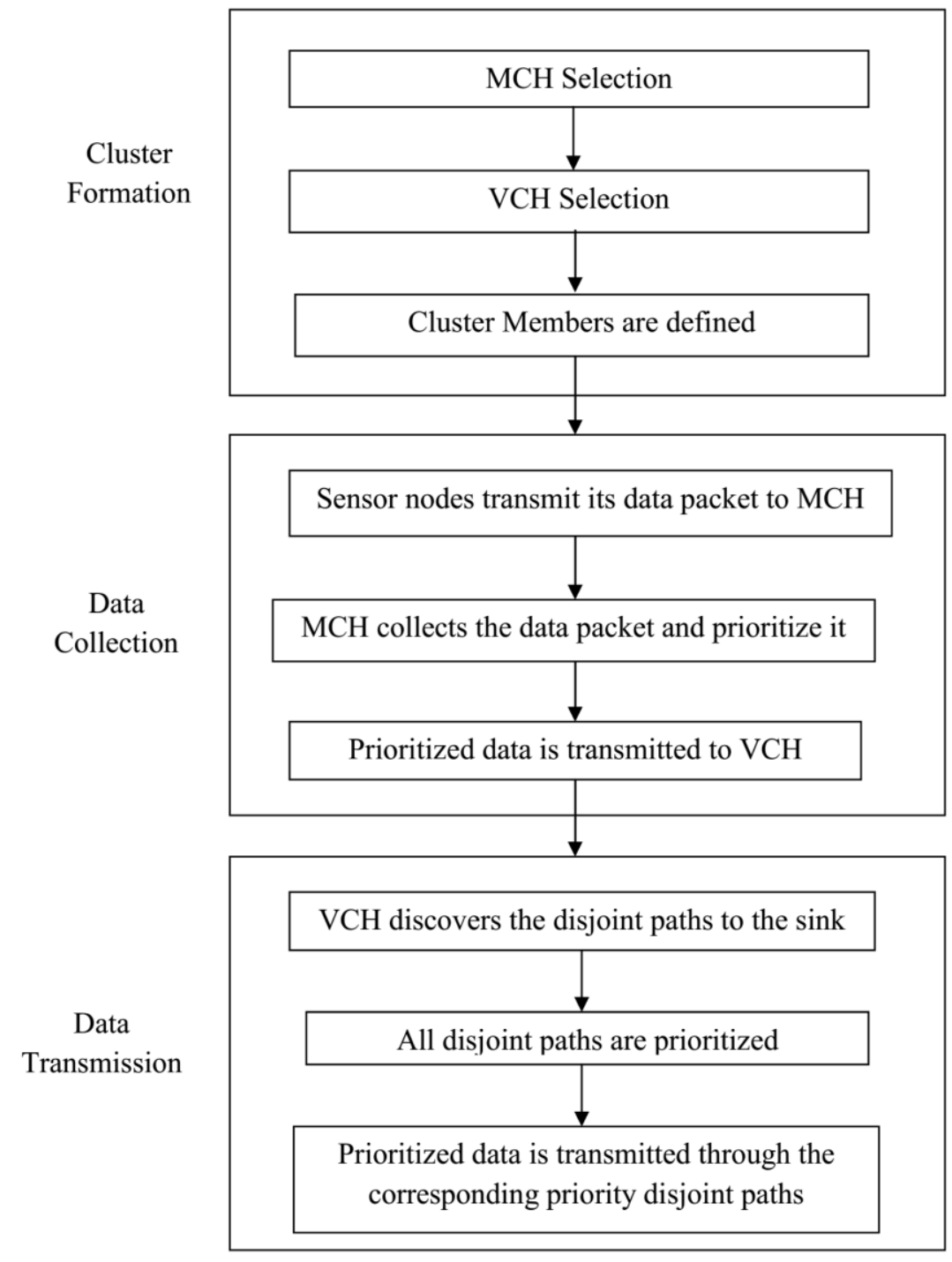

Figure.1 Block Diagram 
Main cluster heads are chosen based on load and link quality in addition to the residual energy. The vice cluster heads are chosen by the main cluster heads when main cluster heads are overloaded or failed and are used to forward the data. These vice cluster heads unload part of energy consumption of main cluster heads.

Then we follow disjoint multipath routing for transmission of multimedia packets [5] which do not have any common node or link so as to provide reliable and efficient data transmission. A queuing system model is formulated based on multimedia packet priorities, which depend on real time and reliability. The traffic classes are categorized into delay-sensitive, loss sensitive, Normal (Delay-andloss insensitive) and Critical (delay and loss sensitive). Alternative disjoint paths are chosen so that packets with different priorities are transmitted based on their conditions. After multiple paths are discovered, they are classified into delay sensitive, Loss sensitive, Critical and Normal, depending on which the corresponding traffic class is selected for transmission.

\subsection{Cluster Formation}

The network model is developed based on some assumptions such that the network location is fixed with limited area, network consists of $\mathrm{N}$ sensor nodes, the distribution of nodes are random, the sensor nodes are immobile, the sink node is fixed and the base station is always situated at the centre of the network [13].

The cluster is formed by the set of nodes which are within each others communication range. To manage all the operations within the cluster, two cluster heads are selected [12]. Initially, the Main Cluster Head $(M C H)$ is selected based on load, link quality and residual energy. Then the Vice Cluster Head $(V C H)$ is selected by the $M C H$. The $M C H$ performs data collection and fusion. The $\mathrm{VCH}$ performs the function of data transmission. The $\mathrm{VCH}$ is used to reduce the overall load on the $\mathrm{MCH}$, and thus decrease the amount of energy consumed. The cluster formation process is described in algorithm 1 .

\section{Algorithm 1}

\section{Notations}

1. Info_Msg : Information Message

2. $R E \quad:$ Residual Energy

3. $R E_{\text {avg }}$ : Average Residual Energy

4. $i \quad$ : sensor node
5. $n$

6. $d_{i}$

: integer value

7. Link $_{\text {cap }}$

: distance from node i

8. Link total

9. Link $_{\text {used }}$

10. $L$

11. $p$

12. $P_{k}$

13. $D_{k}$ by node $\mathrm{k}$

14. $f_{k^{n} k^{\prime}}^{p}$

: Link Capacity

: Total link Capacity

: Used Link Capacity

: Load on node

: link

: node capacity of $\mathrm{k}$

: rate at which packets are generated wireless link $\left(k^{\prime \prime}, k^{\prime}\right)$

15. $k^{\prime \prime}, k^{\prime} \quad:$ links

16. Link $k_{\text {Thres }}$ : Threshold value of Link capacity

17. L L Thres $\quad$ : Load Threshold

18. $t_{1}, t_{2} \quad$ : time

19. $T_{\text {total }} \quad$ : total time in the time interval

20. Cont_Msg : Contending node message

21. $V \quad: \mathrm{VCH}$ selection factor

22. $\mathrm{VCH}$ : Vice Cluster Head

23. $M C H \quad$ : Main Cluster Head

\section{Algorithm}

1. Every sensor node in the network maintains a Neighbor Table in which information related to all of its neighboring nodes are recorded and stored.

2. Initially, each node broadcasts an Info_Msg to all the nodes within a radius of $r$.

3. The Info_Msg consists of two information: the node ID and its $R E$.

4. When a node receives an Info_Msg from its neighbors, it updates its neighbor table after retrieving the data from the received message.

5. Thus, the neighbor table of all the nodes is completely updated with the information of the surrounding nodes.

6. Then, each node calculates the average residual energy of its neighbors based on the distance as given in equation (1)

$$
R E_{\text {avg }}=\sum_{i=1}^{n}\left[R E \cdot \frac{\sum_{i=1}^{n} \frac{d_{i}}{n}}{d_{i}}\right] / n
$$

7. Each node calculates its link capacity, Link $k_{c a p}$ according to equation (2)

$$
\text { Link }_{\text {cap }}=\text { Link }_{\text {total }}-\text { Link }_{\text {used }}
$$

8. Then every node calculates its load, $L$ using equation (3) 


$$
L=\sum_{p=1}^{\left(p_{k}+D_{k}\right) / 2} f_{k^{\prime \prime} k^{\prime}}^{p}
$$

9. Every node compares its Link $k_{\text {cap }}$ with Link $k_{\text {Thres }}$ and $L$ with the $L_{\text {Thres }}$.

10. If $\operatorname{Link}_{\text {cap }}>$ Link $k_{\text {Thres }}$ and $L<L_{\text {Thres, }}$ then it is eligible to compete for the role of $M C H$.

11. After estimating the $\mathrm{RE}_{\mathrm{avg}}$, $\mathrm{Link}_{\mathrm{cap}}$ and $L$, each eligible node broadcasts a Cont_Msg to its neighbors either at time $t_{1}$ or $t_{2}$ given by equation (4) and (5)

$$
\begin{gathered}
t_{1}=\frac{T_{\text {total }}}{2} \times \frac{R E_{\text {avg }}}{R E} \times \sigma, R E_{\text {avg }} \leq R E \\
t_{2}=T_{\text {total }}-\frac{T_{\text {total }}}{2} \times \frac{R E_{\text {avg }}}{R E}, R E_{\text {avg }} \triangleright R E
\end{gathered}
$$

12. Equation (4) represents the nodes sending the Cont_Msg within the time interval $\left[0, T_{\text {total }} 2\right]$ and considered as strong contenders for the role of $\mathrm{MCH}$.

13. Equation (5) represents the nodes sending the Cont_Msg in the time interval $\left[T_{\text {total }} / 2, T_{\text {total }}\right]$ and are considered as weak contenders as the possibility of the message being received by the neighboring nodes within $T_{\text {total }}$ is low.

14. When broadcasting the Cont_Msg, the nodes record the broadcasting time either $t_{1}$ or $t_{2}$.

15. If the node did not receive any message from the surrounding nodes, then it broadcasts its message and then declares itself as the $\mathrm{MCH}$.

16. After the selection of the $M C H$, the $V C H$ are selected by the $M C H$.

17. For the selection of $V C H$, the $M C H$ considers the remaining eligible nodes based on the node $R E$ and node distance from the $M C H$ using the equation (6).

$$
V=\frac{R E}{k \cdot d_{i}}
$$

18. The node at minimum distance from $M C H$ and with maximum $R E$ i.e., node with higher $V$ value is selected as $\mathrm{VCH}$.

19. All the sensor nodes within the transmission range of the $\mathrm{MCH}$ and $\mathrm{VCH}$ are considered as the members of the cluster.

After the selection of the $M C H$ and $V C H$, all the member sensor nodes are monitored continuously by $M C H$ and $V C H$. Thus, $M C H$ and $V C H$ jointly control the cluster operation.

\subsection{Priority Based Multipath Routing}

During communication, the data from the member nodes is transferred to the $\mathrm{MCH}$ which performs data collection and fusion, and then $\mathrm{MCH}$ passes the data to $\mathrm{VCH}$, which performs the task of data transmission [5]. Since the sink node is fixed, the clusters around the sink node are considered as layers. The VCH in every cluster is given a layer ID, with the $\mathrm{VCH}$ closer to the sink being assigned the lowest LID and the LID value increases with the increase in distance from the sink node. The data transmission process is described in algorithm 2 .

\section{Algorithm 2}

\section{Notations}

1. $\mathrm{MCH}$

2. $\mathrm{VCH}$

3. $L I D$

4. $L_{p}$

5. $L_{\text {Thres }}$

6. $L R_{p}$

7. $L R_{\text {Thres }}$

Main Cluster Head

Vice Cluster Head

Layer Identification

end to end latency of path $\mathrm{P}$

Threshold Latency

Loss Rate of path $\mathrm{P}$

Threshold Loss Rate

\section{Algorithm}

1. When a member node needs to transmit data packet to the sink node, it initially sends its data with the sink address to the $M C H$.

2. In the $M C H$, the data is cached in the buffer queue, and then passed into the priority scheduler in a consecutive order.

3. The priority scheduler classifies the incoming data into four groups based on the delay tolerance and loss tolerance level and then prioritizes them.

i) High Priority data: Data that is real time and loss intolerant are of high priority. Example: packets containing alert information or intra frames in a video stream.

ii) Normal Priority Data: Data that is real time but is loss tolerant are of medium priority. Example: packets containing the predictor frames or bidirectional frames.

iii) Low Priority Data: Data that is not real time and loss intolerant are of low priority. Example: critical monitoring processes for offline posting.

iv) Very Low Priority Data: Data that is not real time and loss tolerant are of very low priority. Example: video for storage or offline posting.

4. Then the $M C H$ transmits the prioritized data to the $\mathrm{VCH}$.

5. In the $\mathrm{VCH}$, the prioritized data is cached in the buffer queue.

6. On receiving the prioritized data, the $\mathrm{VCH}$ determines the sink node address to which the data has to be delivered. 
7. On determining the sink node, the $\mathrm{VCH}$ tries to locate the sink node within its communication range.

8. If the $\mathrm{VCH}$ is able to locate the sink node, then it sets up a disjoint path directly.

9. If the $V C H$ is not able to locate the sink node within its communication range, then the $\mathrm{VCH}$ locates another $V C H$ with lower $L I D$ and selects it as the next hop node in the disjoint path.

10. If the $V C H$ is unable to locate the sink node and also a $V C H$ with lower $L I D$, then the $V C H$ expands its transmission range until it finds either the sink node or another $\mathrm{VCH}$.

11. After discovering various disjoint paths towards the sink node, the $\mathrm{VCH}$ categorizes the path based on delay tolerance and loss tolerance.

12. For each path, $\mathrm{P}$ between two $\mathrm{VCH}$, the $\mathrm{VCH}$ estimates the end to end latency, $L_{p}$ and loss rate, $L R_{p}$ and compares it with the predefined threshold latency, $L_{\text {Thres }}$ and threshold loss rate, $L R_{\text {Thres. }}$.

i) If $L_{P}>L_{\text {Thres }}$ then the path is considered as delay sensitive.

ii) If $L R_{p}>L R_{\text {Thres }}$ then the path is considered as loss sensitive.

iii) If $L_{P}>L_{\text {Thres }}$ and $L R_{p}>L R_{\text {Thres }}$, then the path is considered as delay and loss sensitive i.e., this is a critical path.

iv) If $L_{P}<L_{\text {Thres }}$ and $L R_{p}<L R_{\text {Thres }}$, then the path is considered as delay and loss insensitive i.e., this is a normal path.

13. After the categorization of the disjoint paths, the prioritized data in the buffer queue of the $\mathrm{VCH}$ is passed into the routing scheduler, where each priority data is moved into the corresponding disjoint path.

i) High priority data is transmitted through the critical path.

ii) Medium priority data is transmitted through the delay sensitive path.

iii) Low priority data is transmitted through the loss sensitive path.

iv) Very low priority data is transmitted through the normal path.

Thus the data is transmitted through the disjoint path based on its priority level and delivered successfully at the sink node.

\section{Simulation Results}

\subsection{Simulation Parameters}

We use NS-2 to simulate our proposed Cluster based Multipath Routing for Wireless Multimedia Sensor Networks (CBMRP) protocol. We use the
IEEE 802.11 for wireless sensor networks as the MAC layer protocol. It has the functionality to notify the network layer about link breakage. In our simulation, the packet sending rate is varied as 100 , $150,200,250$ and $300 \mathrm{~Kb}$

Table 1. Simulation parameters

\begin{tabular}{|l|l|}
\hline No. of Nodes & 100 \\
\hline Area & $500 \times 500 \mathrm{~m}$ \\
\hline MAC Protocol & 802.11 \\
\hline Simulation Time & $50 \mathrm{sec}$ \\
\hline Traffic Source & CBR and Exponential \\
\hline Rate & $\begin{array}{l}100,150,200,250 \quad \text { and } \\
300 \mathrm{~Kb}\end{array}$ \\
\hline Propagation & TwoRayGround \\
\hline Antenna & OmniAntenna \\
\hline Initial Energy & $10.1 \mathrm{~J}$ \\
\hline $\begin{array}{l}\text { Transmission } \\
\text { Power }\end{array}$ & 0.660 \\
\hline Receiving Power & 0.395 \\
\hline
\end{tabular}

The area size is 500 -meter $\mathrm{x} 500$-meter square region for 50 seconds simulation time. The simulated traffic is Constant Bit Rate (CBR) and Exponential (Exp).

Our simulation settings and parameters are summarized in table 1.

\subsection{Performance Metrics}

We evaluate performance of the new protocol mainly according to the following parameters. We compare the EEPCA [11] protocol with our proposed CBMRP protocol.

Average Packet Delivery Ratio: It is the ratio of the number of packets received successfully and the total number of packets transmitted.

Average end-to-end delay: The end-to-enddelay is averaged over all surviving data packets from the sources to the destinations.

Packet Drop: It is the number of packets dropped during the data transmission

\subsection{Results and Analysis}

We vary the data transmission rate as 100,150 , 200.250 and $300 \mathrm{~Kb}$ for both $\mathrm{CBR}$ and VBR (Exponential) traffic. 


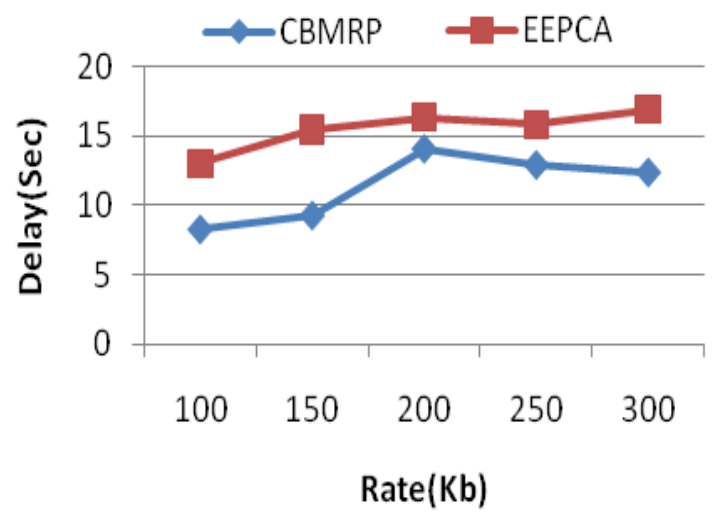

Figure.2 Rate Vs Delay

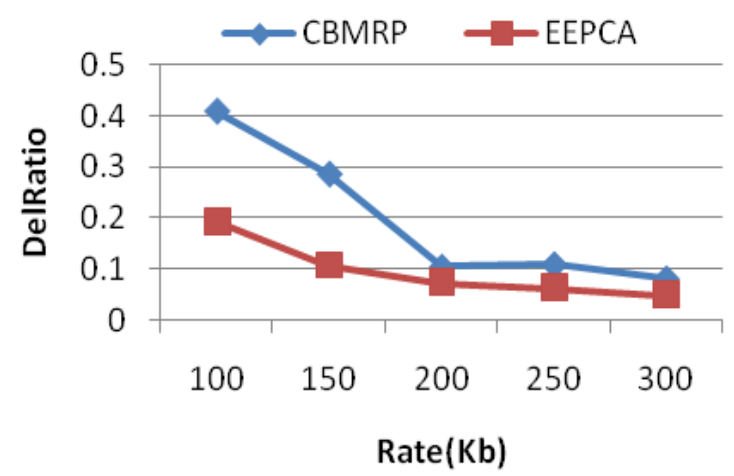

Figure.3 Rate Vs Delviery Ratio

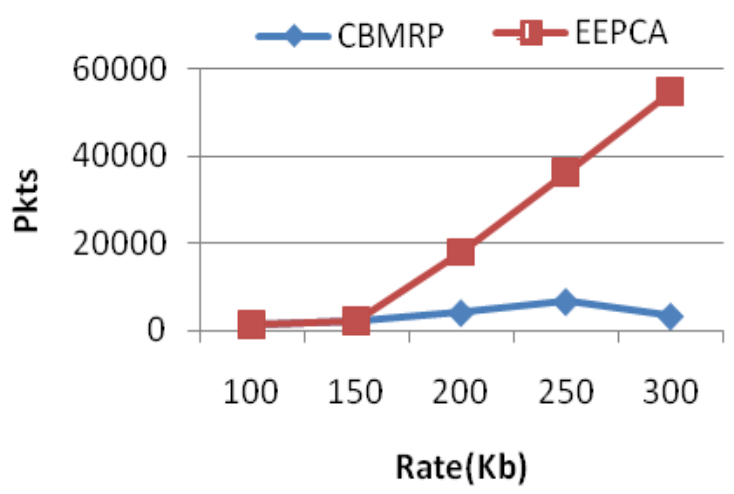

Figure.4 Rate Vs Drop

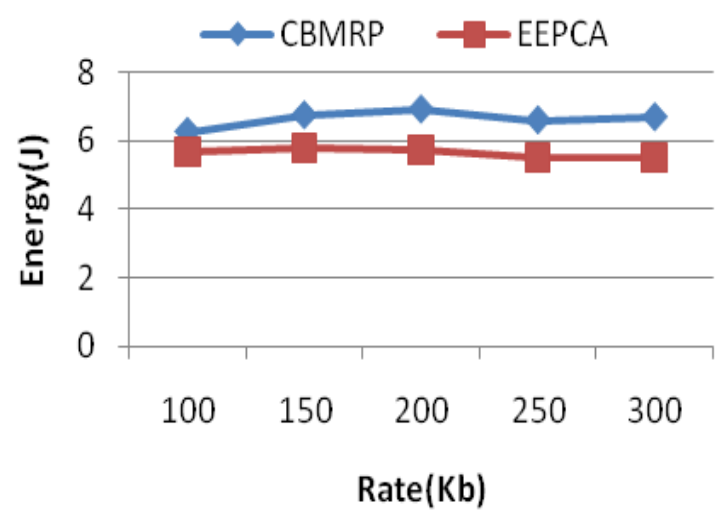

Figure.5 Rate Vs Enregy

\section{Case-1 Results for CBR Traffic}

Figures 2 to 5 show the results of delay, delivery ratio, packet drop, and energy consumption by varying the rate from $100 \mathrm{~Kb}$ to $300 \mathrm{~Kb}$ for the $\mathrm{CBR}$ traffic in CBMRP and EEPCA protocols. When comparing the performance of the two protocols, we infer that CBMRP outperforms EEPCA by $27 \%$ in terms of delay, $45 \%$ in terms of delivery ratio, $53 \%$ in terms of packet drop, and $15 \%$ in terms of energy consumption.

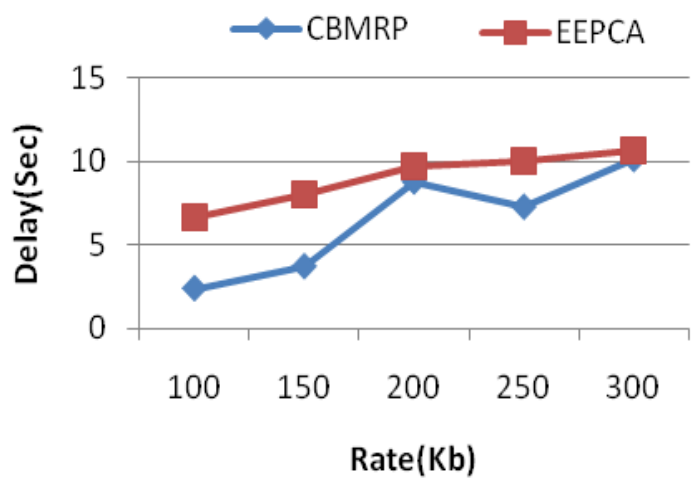

Figure.6 Rate Vs Delay

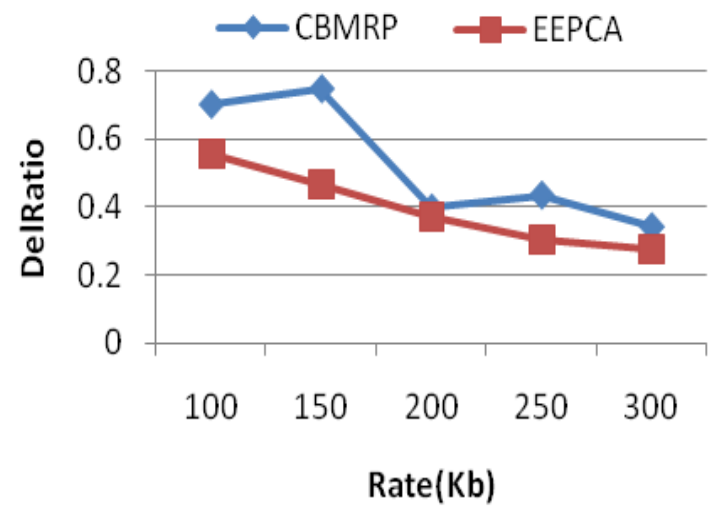

Figure.7 Rate Vs Delivery Ratio

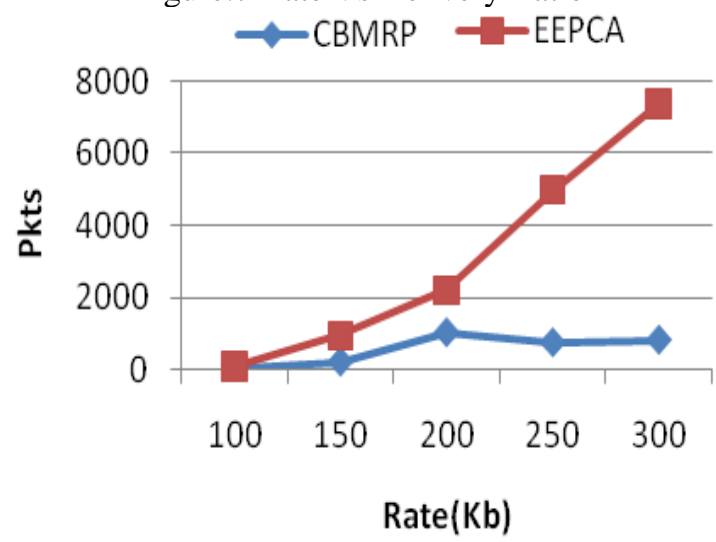

Figure.8 Rate Vs Drop 


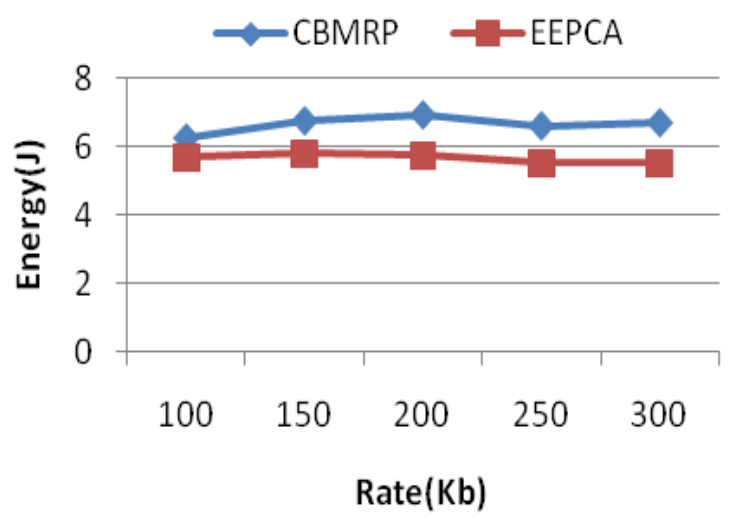

Figure.9 Rate Vs Energy

\section{Case-2 Results for VBR Traffic}

Figures 2 to 5 show the results of delay, delivery ratio, packet drop, and energy consumption by varying the rate from $100 \mathrm{~Kb}$ to $300 \mathrm{~Kb}$ for the EXP traffic in CBMRP and EEPCA protocols. When comparing the performance of the two protocols, we infer that CBMRP outperforms EEPCA by $32 \%$ in terms of delay, $23 \%$ in terms of delivery ratio, $71 \%$ in terms of packet drop, and $15 \%$ in terms of energy consumption.

\section{Conclusion}

In this paper, we have developed an efficient technique for Cluster based Multipath Routing in Wireless Multimedia Sensor Networks. Initially, a cluster is formed with two cluster heads: the Main Cluster Head (MCH) and Vice Cluster Head (VCH). These two CHs collectively handle the cluster operation by dividing the tasks between them. The $\mathrm{MCH}$ collects the data to be transmitted from the member sensor nodes and prioritizes them based on delay tolerance and loss tolerance level. Then the prioritized data is transmitted to the $\mathrm{VCH}$. The $\mathrm{VCH}$ determines all the possible disjoint paths towards the sink node and prioritizes the path based on delay tolerance and loss rate. Then the data is transmitted to the sink through the corresponding priority disjoint path. Thus, the data is successfully delivered at the sink nodes according to the real time need. In addition to this in future, we planned to propose fuzzy network for cluster formation and routing purpose. So that delay can be improved and energy, delivery ratio everything is improved based on the proposed method

\section{References}

[1] J. Agrakhed, G. S. Biradar and V. D. Mytri, "A New QoS Adaptive Multipath Routing in Wireless
Multimedia Sensor Network", Fourth International Conference on Computational Intelligence and Communication Networks, pp. 66-72, 2012.

[2] J. Agrakhed, G. S. Biradar and V. D. Mytri, "Adaptive Multi Constraint Multipath Routing protocol in Wireless Multimedia Sensor Network", International Conference on Computing Sciences, pp. 326-331, 2012.

[3] Z. Bidai and M. Maimour, "Multipath Routing for High-Data Rate Applications in ZigBee Wireless Sensor Networks", IEEE 6th International Conference on New Technologies, Mobility and Security (NTMS), pp. 1-5, 2014.

[4] G. Liu and C. Wei, "A New Multi-Path Routing Protocol Based on Cluster for Underwater Acoustic Sensor Networks", International Conference on Multimedia Technology (ICMT), pp. 91-94, 2011.

[5] Q. Ye, M. Wu and Y. Wang, "Traffic Scheduling Scheme for Disjoint Multipath Routing Based Wireless Multimedia Sensor Networks", IEEE AsiaPacific Services Computing Conference, pp. 388-393, 2010.

[6] Y. Jing, X. Mai, X. Jinfu, X. Baoguo and H. Lu, “A Cluster-Based Multipath Delivery Scheme for Wireless Sensor Networks", Proceedings of ICBNMT, pp. 286-291, 2009.

[7] H. Huang, X. Cao, R. Wang and L. Sun, “A Novel Clustering Ant-based QoS-aware Routing Algorithm in Large Scale Wireless Multimedia Sensor Networks", IEEE International Conference on Cluster Computing Workshops, pp. 184-191, 2012.

[8] S. Lohier, A. Rachedi and Y. G. Doudane, "A cost function for QoS-aware routing in Multi-Tier Wireless Multimedia Sensor Networks", WiredWireless Multimedia Networks and Services Management. Springer Berlin Heidelberg, pp. 81-93, 2009.

[9] Y. Sun, H. Ma, L. Liu and Y. Zheng, "ASAR: An antbased service-aware routing algorithm for multimedia sensor networks", Front. Electr. Electron. Eng. China, Vol. 3, No. 1, pp. 25-33, 2008.

[10] X. Li, W. Gang, L. Zongqi and Z. Yanyan, “An energy-efficient routing protocol based on particle swarm clustering algorithm and inter-cluster routing algorithm for WSN", 25th Chinese Control and Decision Conference (CCDC), pp. 4029-4033, 2013.

[11] J. Yua, Y. Qi, G. Wang and X. Gu, “A cluster-based routing protocol for wireless sensor networks with non uniform node distribution", International Journal of Electronics and Communications, Vol. 66, No. 1, pp. 54-61, 2012.

[12] F. Li, X. Wang and T. Xu, "Energy-aware Data Gathering and Routing Protocol Based on Double Cluster-heads", Communications in Information Science and Management Engineering, PP. 40594063, 2011.

[13] J. Peng, T. Liu, H. Li and B. Guo, "Energy-Efficient Prediction Clustering Algorithm for Multilevel Heterogeneous Wireless Sensor Networks", Hindawi 
Publishing Corporation, International Journal of Distributed Sensor Networks, Vol. 4, pp. 1-17, 2013.

[14] M. A. Alim, Y. Wu and W. Wang, "A Fuzzy Based Clustering Protocol for Energy-efficient Wireless Sensor Networks", Proceedings of the 2nd International Conference on Computer Science and Electronics Engineering (ICCSEE 2013), Vol. 760, pp. 685-690, 2013.

[15] B. S. Mathapati, S. R. Patil and V. D. Mytri, "A Cluster Based Energy Efficient Reliable Routing Protocol for Wireless Sensor Networks", 1st International Conference on Emerging Technology Trends in Electronics, Communication and Networking, pp. 1-6, 2012

[16] Network Simulator: http:///www.isi.edu/nsnam/ns 